\title{
Prävention der Homophobie: Was bereits getan wurde und was noch zu tun ist
}

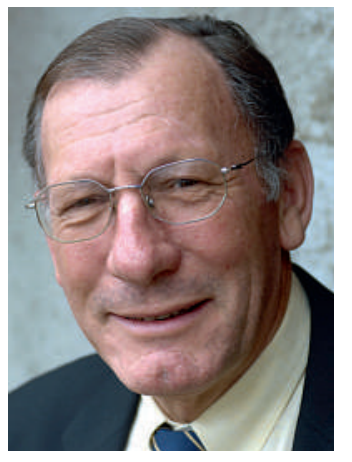

Jean Martin

Literatur

1 Thorens-Gaud E. Adolescents homosexuels - Des préjugés à l'acceptation. Lausanne: Editions Favre; 2009.

2 Nau JY. De quel genre êtes-vous: «pro-inné» ou «pro-acquis»? (à propos d'identité de genre). Revue médicale suisse. 2011; 7:2202-3.

3 Das Tabu wird bei homoparentalen Familien langsam aufgehoben - zum einen in Bezug auf die Tatsache, dass es solche Familien gibt, und zum anderen bezüglich der Erkenntnis, dass die solchen Familien entstammenden Kinder nicht notwendigerweise ein gestörtes Verhalten aufweisen. Bislang letzter frankophoner Beweis: Carrière Z, Tervonen T. Fils de. Paris: Editions Trans; 2011 (s. Le Temps, Genève, 8 novembre 2011, p. 28)
Anfang November dieses Jahres fanden in Lausanne die PREOS-Tage (Journées romandes sur la prévention du rejet basé sur l'orientation sexuelle et l'identité de genre chez les jeunes) statt, mit besonderer Berücksichtigung des schulischen Milieus. Unterstützt wurde die Initiative vom Bundesamt für Gesundheit, zwei Kantonen und acht grossen westschweizerischen Städten. Vier Staatsrätinnen und Staatsräte haben das Wort ergriffen und sich dafür ausgesprochen, dass die Prävention der Homophobie in den Unterrichtsstoff aufgenommen werden solle. Bill Ryan von der Universität McGill in Montréal machte deutlich, wie der Kampf auf diesem Gebiet in Kanada effizient geführt wird.

Dabei bietet der Blick auf die bereits zurückgelegte Wegstrecke Interessantes. In den 80ern war einer meiner Kantonsarzt-Kollegen bereit, den Ausschluss von Homosexuellen aus dem Lehrkörper zu empfehlen (unabhängig davon, ob fragwürdige Handlungen vorlagen oder nicht). In den 90ern fragte mich PierreAndré Michaud, Leiter der Unité de Santé des Adolescents des CHUV einmal, ob wir das «Risiko eingehen» und einem jungen Homophilen empfehlen könnten, die Jugendgruppe VoGay (damals neu gegründeter waadtländischer Verein für Homosexuelle) zu kontaktieren, um über sein Problem zu sprechen. Wir haben entschieden, den Schritt zu wagen. Gleichzeitig war uns aber bewusst, dass wir uns gegebenenfalls vor Eltern zu verantworten haben würden, die eine offizielle Dienststelle beschuldigen, ihren Jungen «in die Lasterhaftigkeit» getrieben zu haben. In diesem Kontext ist an das starke Engagement von Homosexuellengruppen in der Prävention von HIV/Aids in einer Zeit zu erinnern, als die Medizin relativ machtlos war, sowie an die konstruktive Zusammenarbeit mit der öffentlichen Gesundheit.

Ich gestehe, dass ich, als ich Anfang der 90er von Homosexuellen gebeten wurde, sie dabei zu unterstützen, vor Schulklassen über Homosexualität sprechen zu dürfen, gesagt habe, dass ich mir dies zwar für die Zukunft vorstellen könne, es zum damaligen Zeitpunkt jedoch für illusorisch hielt. Heute gibt es solche Veranstaltungen in deutschsprachigen Kantonen. Ende 2010 hat mich die Qualität des Wandels überrascht, als ich erfuhr, dass die Erziehungsdirektionen der Kantone Genf und Waadt eine Delegierte für Fragen zur Homosexualität (die ein Buch über ihre Lehrerfahrung veröffentlicht hat [1]) ernannt haben. In einem Zeitraum von zwanzig Jahren sind wir von einem genierten Schweigen zu diesem Thema zu einem expliziten Engagement der öffentlichen Entscheidungsträger gegen die Homophobie gekommen - oft im weiter gefassten Rahmen der Menschen- rechte und des Kampfes gegen jegliche - auch rassistische - Art von Diskriminierung. In Genfer Schulen findet sich die Plakatierung: «Hier sprechen wir über sexuelle Vielfalt».

Hauptpunkt: Die Anerkennung - insbesondere seitens der Medizin - dass Homosexualität im Wesentlichen nicht Ergebnis einer Entscheidung ist (selbst wenn Kontextfaktoren in zwiespältigen Situationen eine Rolle spielen können). Daher das Bedürfnis, sich eindeutig von jenen Stimmen zu distanzieren, die immer noch behaupten, es handle sich um Krankheit oder Sünde! Vor diesem Hintergrund waren die Gay Prides für Homosexuelle und im weiteren Sinne für die LGBT (Lesben, Homosexuelle, Bisexuelle und Transgender) ein Medium, um ihr Recht auf Anderssein zu bestätigen, wenngleich gelegentlich auf provokante Art und Weise. «Ich habe das Recht, aussen das zu sein, was ich innen bin», zitiert Bill Ryan.

Unsere Gesellschaft und ihr Rechtsrahmen sind gleich in mehrerlei Hinsicht gefordert. Selbst unter blosser Berücksichtigung wissenschaftlicher und sozialer Fakten stellt der jüngst vom Bundesgericht bestätigte Entscheid, dass die Partner gleichgeschlechtlicher Paare die Kinder des anderen Partners nicht adoptieren dürfen, eine Diskriminierung dar. Es ist wahr, dass die Wissensentwicklung in vielen Fällen an alten Positionen rüttelt (in einigen Fällen auch an meinen), aber selbst wenn wir aus Glaubensgründen oder weil es politisch opportun ist, die Entwicklung verlangsamen wollten, könnte die Gesellschaft die Realität nicht ignorieren. «Fakten sind stur.»

Die katholische Kirche macht geltend, dass «männlich und weiblich ontologisch Teil der Schöpfung sind» [2]. Ja, aber dies bietet keine Legitimierung dafür, dass die Tatsache nicht zur Kenntnis genommen wird, dass sich eine bestimmte Anzahl unserer Mitmenschen nicht in eine rigide, objektiv vereinfachte Kategorisierung einpassen lässt. Ein Rechtsstaat muss die Grundrechte des Individuums respektieren. Wir haben zwar ein Recht auf Überzeugungen, doch es gibt ein gesellschaftliches und staatsbürgerliches Grundproblem, wenn irrige Überzeugungen gegen besseres Wissen in den für uns alle geltenden Gesetzen aufrechterhalten werden [3]. Mit Artikel 261 bis StGB verfügt die Schweiz zwar über eine Antirassismus-Regelung, doch es gibt keinen gleichlautenden Text für andere Diskriminierungen, inklusive Diskriminierung der sexuellen Orientierung. Mehrere Redner der PREOS-Tage wünschen sich eine solche Entwicklung.

Jean Martin, Mitglied der nationalen Ethikkommission und der Redaktion 